\title{
The Arabidopsis Class VIII Myosin ATM2 Is Involved in Endocytosis
}

\author{
Amirali Sattarzadeh, Rainer Franzen, and Elmon Schmelzer* \\ Max-Planck-Institute for Plant Breeding Research, Central Microscopy, \\ Cologne, Germany
}

\begin{abstract}
Members of the class XI of the myosin superfamily comprising higher plant, actin-based molecular motors have been shown to be involved in peroxisome and Golgi vesicle trafficking comparable to yeast and animal class $\mathrm{V}$ myosins. The tasks of the second class of myosins of higher plants, class VIII, are unclear. In this study the class VIII myosin ATM2 from the model plant Arabidopsis thaliana was selected for the examination of cargo specificity in vivo. Fluorescent proteinfusion plasmid constructs with fragments of the ATM2 cDNA were generated and used for Agrobacterium tumefaciens-based transient transformation of Nicotiana benthamiana leaves. The resulting subcellular localization patterns were recorded by live imaging with confocal laser scanning microscopy (CLSM) in epidermal leaf cells. Expression of a nearly full-length construct displayed labeling of filaments and vesicles, a head + neck fragment led to decoration of filaments only. However, expression of fluorescent protein-tagged C-terminal tail domain constructs labeled vesicular structures of different appearance. Most importantly, coexpression of different RFP/YFP-ATM2 tail fusion proteins showed colocalization and, hence, binding to the same type of vesicular target. Further coexpression experiments of RFP/YFP-ATM2 tail fusion proteins with the endosomal marker FYVE and the endosomal tracer FM4-64 demonstrated colocalization with endosomes. Colocalization was also detected by expression of the CFP-tagged membrane receptor BRI1 as marker, which is constantly recycled via endosomes. Occasionally the ATM2 tail targeted to sites at the plasma membrane closely resembling the pattern obtained upon expression of the YFP-ATM1 C-terminal tail. ATM1 is known for its localization at the plasma membrane at sites of plasmodesmata. Cell Motil. Cytoskeleton 65: 457-468, 2008. (c) 2008 Wiley-Liss, Inc.
\end{abstract}

Key words: plant class VIII myosins; cargo binding; endocytosis; fluorescent protein tagging; life imaging

\section{INTRODUCTION}

Endocytosis in plants has recently gained increasing attention because of the finding that receptors can be internalized similar to animals. This holds true for receptors recognizing endogenous signals such as brassinosteroid hormones [Russinova et al., 2004] or not yet defined molecules involved in development [Gifford et al., 2005] as well as exogenous signals such as pathogen-associated molecular patterns in plant innate immunity [Robatzek et al., 2006] Internalization of the receptors of the endogenous signals is constitutive. However, the best characterized paradigm of plant immune receptors, the FLS2 receptor kinase, which recognizes bacterial flagellin and triggers the defence response [Gomez-Gomez and Bol-
Contract grant sponsor: Deutsche Forschungsgemeinschaft (DFG).

Amirali Sattarzadeh's present address is Department of Molecular Biology and Genetics, Biotechnology Bldg., Cornell University, Ithaca, New York.

*Correspondence to: Elmon Schmelzer, Max-Planck-Institute for Plant Breeding Research, Central Microscopy, Carl-von-Linné-Weg 10, 50829 Cologne, Germany. E-mail: schmelze@mpiz-koeln.mpg.de

Received 24 October 2007; Accepted 13 February 2008

Published online 7 April 2008 in Wiley InterScience (www. interscience.wiley.com).

DOI: $10.1002 / \mathrm{cm} .20271$ 
ler, 2002], is induced to be internalized specifically upon ligand binding. This is the first example of ligand-mediated receptor endocytosis in plants [Robatzek et al., 2006]. Previously, nothing was known about the further degradation or potential recycling of these receptors and the route and processing of the according endosomal vesicles. Although knowledge of endosomal trafficking in plants lags far behind animal and yeast, the major vesicular compartments of the endocytic pathway have been described [Geldner, 2004; Samaj et al, 2005]. Resembling the situation in animal cells, uptake of material happens through clathrin-coated pits which are processed to early endosomes, sometimes called partiallycoated reticulum (PCR). The next stage comprises the late endosomes/multivesicular bodies/ prevacuolar compartment and is connected to the vacuole and lysosomes and to the trans-Golgi network, which is thought to participate in extensive exchange of material.

In animal cells, several actin-based molecular motors of the large family of myosins, myosin II, Va, VI and VIIa are required for the transportation of endosomes [Buss et al., 2001; Aschenbrenner et al., 2003; Holt et al., 2007]. This raises the possibility that myosins might also be involved in plant endocytosis. In higher plants, the myosins exclusively fall into two classes, class XI and class VIII [Hodge and Cope, 2000; Berg et al., 2001]. In the genome of the model plant Arabidopsis thaliana 17 myosin genes were identified, 4 of them belong to class VIII and 13 to class XI [Reddy and Day, 2001]. Although plant myosins appear to form a clade of their own, the available sequence data nevertheless suggest that they follow the domain pattern typical for most of the myosins: the highly conserved $\mathrm{N}$-terminal head (motor) domain responsible for ATP hydrolysis, binding to actin and production of force, the neck domain containing characteristic repeat motifs (IQ repeats), and the C-terminal tail domain mediating cargo binding. The IQ motifs of the neck domain are known to be binding sites for calmodulin or regulatory light chains in other systems, though binding partners for plant myosins are unknown. The tail domain usually contains one or more coiled-coil regions responsible for dimerization.

Recent findings have elucidated the cargo specificity of myosins from A. thaliana belonging to the class XI of the plant myosins [Holweg and Nick, 2004; Wang and Pesacreta, 2004; Hashimoto et al., 2005; Li and Nebenfuhr, 2007; Reisen and Hanson, 2007; Peremyslov et al., 2008; Avisar et al., 2008b]. According to these reports, members of class XI transport organelles such as Golgi stacks and peroxisomes. In animals and fungi organelle movements are driven by the class V myosins. Structural similarity of the cargo-binding globular tail domain was found between this class and the plant class XI myosins [Li and Nebenfuhr, 2007]. This implicates functional analogy of plant class XI myosins to non-plant class V myosins. Little information is available about the second class of higher plant myosins, class VIII. The subcellular localization of ATM1, the first described class VIII myosin from A. thaliana, was investigated by transmission electron microscopy and immunocytology [Reichelt et al., 1999]. In maize root apices labeling with ATM1specific antibodies was detected at the transversal plasma membrane between cells that had just divided, preferentially at plasmodesmata. Further immunolocalization studies indicated relocation of the labeling to the surface of statoliths in maize root cap cells upon hyperosmotic stimulation accompanied by the reorganization of the actin cytoskeleton [Wojtaszek et al., 2005] whereas in A. thaliana leaves the ATM1-specific antibodies decorated chloroplast surfaces. The authors suggest from their data a role of myosin VIII in actin filament anchoring at sites of plasmodesmata and in osmosensing. Recent investigations on class VIII myosins from Nicotiana benthamiana render obvious that class VIII myosins function in protein delivery to plasmodesmata [Avisar et al., 2008a]. In this study ectopic overexpression of the tail domains of class VIII myosins, but not class XI myosins, interferred with the plasmodesmatal targeting of the Hsp70 homolog (Hsp70h) of Beet yellow virus (BYV) essential for virion assembly and cell-to-cell movement. Another recent publication employed GFP tagging technology to investigate the subcellular localization of ATM1 [Golomb et al., 2008]. By transient expression of an ATM1-tail domain GFP fusion protein (GFP::ATM1-IQ tail) in $N$. benthamiana the fluorescent signal was found in pit fields where callose had accumulated, which strongly suggests plasmodesmatal localization. Furthermore, GFP::ATM1-IQ tail colocalized with the endosomal tracer FM4-64 and partially with the endosomal marker ARA6 indicating a role of ATM1 in endocytosis.

In our present study we investigated the subcellular localization and cargo-binding specificity of the class VIII myosin ATM2 and, for comparison, of ATM1 from A. thaliana. We demonstrate that ATM2 binds to vesicles that belong to the endocytotic pathway. Sporadically it was also found at plasmodesmata. ATM1, however, was found at plasmodesmata, in accordance with earlier data [Reichelt et al., 1999; Wojtaszek et al., 2005; Golomb et al., 2008].

\section{MATERIALS AND METHODS RNA Extraction, RT-PCR, and Plasmid Construction}

RNA was extracted from A. thaliana cv Columbia leaves using RNeasy Plant Mini Kit (Qiagen) followed by reverse transcription using Superscript II reverse tran- 
scriptase (Qiagen) with an oligo-dT primer. Myosin VIII constructs from the cDNAs of the genes AT3G19960 (ATM1) and AT5G54280 (ATM2) were amplified by PCR allowing GATEWAY directional cloning using the attB primer extension sites by Accu prime ${ }^{\mathrm{TM}}$ Taq DNA polymerase (Invitogen, Karlsruhe, Germany) to generate PCR products that are flanked by attB sites. The following primers were used:

- ATM2head+IQ-forward TCATGTTGTCCACT GCAAATGTTG

- ATM2head+IQ-reverse CAGACATAGATGTT GGTTGAACT

- ATM2tail-long-forward TCATGCAAAGACAA AAAGAACTACG

- ATM2tail-long-reverse (stop) CTCACCTCTTT TTCCCCACCATTTC

- ATM2tail-long-reverse CCCTCTTTTTCCCCACCATTTC

- ATM2tail-short-forward TCATGTCTGATCTC CAGAAACGGAT

- ATM2tail-short-reverse (stop) CTCATCTATG GAGTCTTGCTTTAGTA

- ATM2head + tail-reverse CCTAGCCTCTTT TTCCCCACCA

- ATM1tail-long-forward TCATGGATATTGGA TGGTTAAAATCTG

- ATM1tail-long-reverse CTCAATACCTGGTGC TATTTCTC

Separate BP recombination reactions with the donor vector $p D O N R .221$ were performed to generate entry clones containing myosin VIII constructs according to the manufacturer's instructions (Invitrogen). The entry clone plasmid DNA was extracted using the Plasmid Isolation Mini Kit (Qiagen, Hilden, Germany) and verified by PCR amplification and sequencing. The LR reaction was used to transfer the insert from entry clones to the destination vector containing the $2 \times \mathrm{CaM} 35 \mathrm{~S}$ promoter plus $Y F P / R F P$ placed N/C-terminally of a Gateway recombination cassette according to the manufacturer's instructions (Invitrogen, Karlsruhe, Germany). The insert sizes were verified by PCR amplification. The expression clone plasmid DNA was extracted using the company's protocol (Qiagen, Hilden, Germany, MachereyNagel, Düren, Germany).

\section{Plant Growth and Transient Expression of Fluorescence Protein Fusion Constructs}

Nicotiana benthamiana. Leaves of 4-6 week old $N$. benthamiana plants grown at $22^{\circ} \mathrm{C}$ under a $16 \mathrm{~h}$ light/ $8 \mathrm{~h}$ dark cycle were used for transformation. Extracted plasmids were electroporated into A. tumefaciens GV3101 carrying the helper plasmid pMP90RK [Koncz et al., 1990] and selected on agar plates containing the appropriate antibiotics. Transformation of $N$. benthamiana leaves by A. tumefaciens directly from plates was performed. Cultures were resuspended in $10 \mathrm{mM} \mathrm{MgCl} 2,10 \mathrm{mM}$ MES $\mathrm{pH} 5.6$ and $150 \mu \mathrm{M}$ acetosyringone to an OD of 0.5 , and incubated for $2-5 \mathrm{~h}$ at $28^{\circ} \mathrm{C}$ in the dark, and infiltrated with a needleless syringe from the abaxial side into $N$. benthamiana leaves. The level and duration of expression was substantially improved by employing coexpression of a virus-encoded suppressor of gene silencing, the p19 protein of tomato bushy stunt virus (TBSV), that prevents post-transcriptional gene silencing (PTGS ) in the infiltrated tissues [Voinnet et al., 2003]. Observations were made 3-5 days after infiltration.

\section{Labeling and Staining of Endosomal Compartments and Nuclei, Plasmolysis}

For visualization of the endosomal compartments, the RFP fusion protein of the tandem FYVE domain, which was recently shown to label plant endosomes, was used [Voigt et al., 2005]. The BRI1-CFP construct [Russinova et al., 2004] was amplified by PCR using the folowing primers: BRI1forward, ATGAAGACTTTTTCAAGCTTCTTT; BRI1reverse, CTAATTTTCCTTCAGGAA. This amplificate was cloned by GATEWAY technology in the destination vector containing the $2 \times$ CaM 35S promoter plus CFP placed C-terminally of a Gateway recombination cassette. For staining with the endosomal tracer FM4-64 (Invitrogen, Karlsruhe, Germany) leaves of $N$. benthamiana were immersed for 15 min in a solution of FM4-64 $(10 \mu \mathrm{M})$ or infiltrated with a needleless syringe. For propidium iodide counter staining of nuclei, leaves were immersed in a solution of $2.5 \%$ mannitol $/ 0.01 \%$ silwet $/ 0.2 \%$ propidium iodide (all chemicals from SIGMA-ALDRICH, Taufkirchen, Germany). For plasmolysis, leaves were mounted with $300 \mathrm{mM}$ $\mathrm{NaCl}$.

\section{Confocal Laser Scanning Microscopy}

CLSM was performed on a Leica DMRE-7 (SDK) microscope equipped with a TCS-SP2 confocal scanning head (Leica Microsystems, Heidelberg, Germany). The $514 \mathrm{~nm}$ line of an argon laser was used to excite YFP and FM4-64, a He/Ne laser at $562 \mathrm{~nm}$ for RFP. The CFP was excited by using a $405 \mathrm{~nm}$ diode laser. Emission of fluorescence was detected in the range of 570-634 nm for RFP constructs and 535-545 nm for YFP constructs. The FM4-64 emission was detected between 600 and $700 \mathrm{~nm}$. Images were recorded and processed using the LCS software 2.5 (Leica Microsytems, Heidelberg, Germany). The imaging in colocalization experiments was done in the sequential mode (line by line) in order to 
A

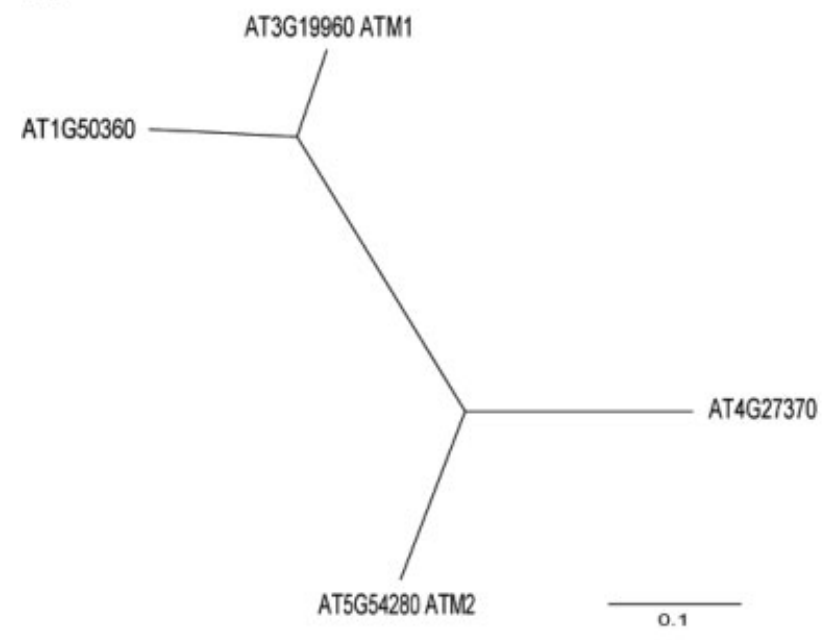

B

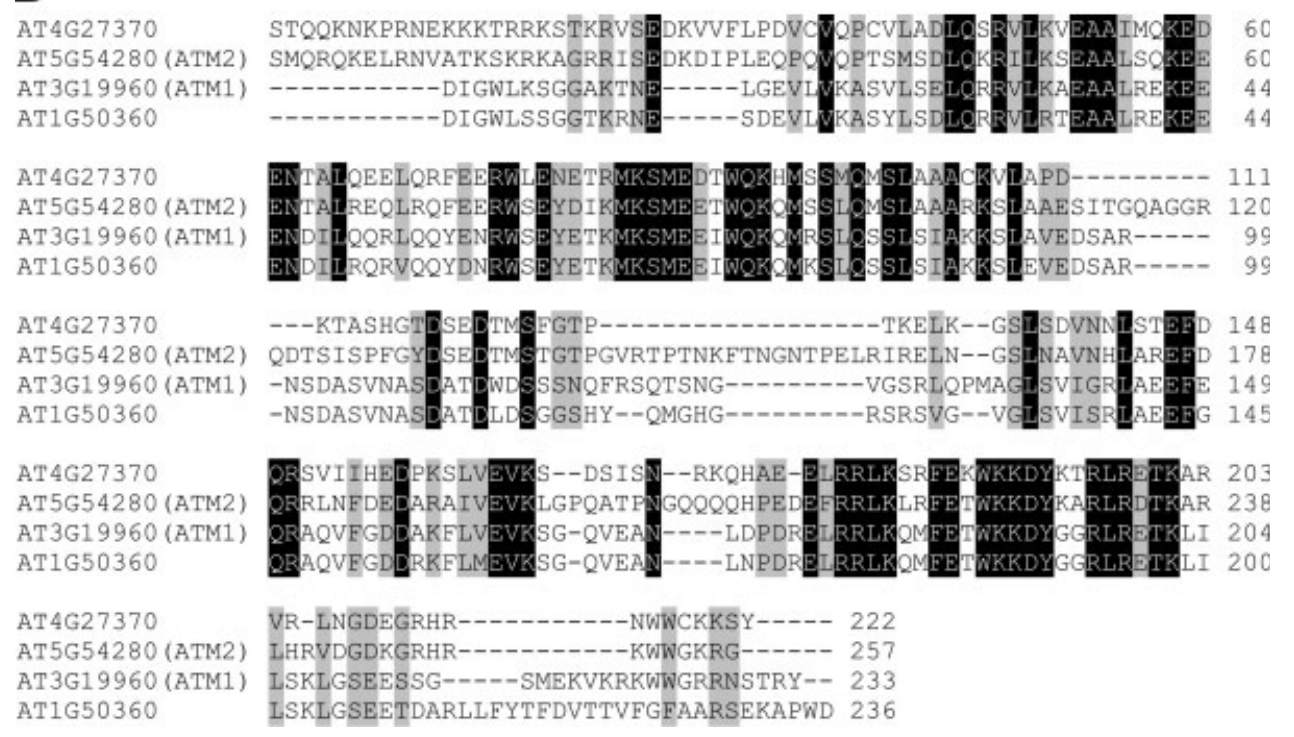

Fig. 1. Cladogram and alignment of the Arabidopsis thaliana class VIII myosin amino acid sequences. A: The cladogram is based on the alignment (Clustalw) of the full-length amino acid sequences and was calculated with the Gonnet 250 matrix. B: The alignment of the four globular tail domains, C-terminally starting with the amino acid after the last IQ repeat, was performed with clustalw (http://www.ebi.ac.uk/ Tools/clustalw/index.html).

exclude cross-talk due to spectral overlap of the fluorophores.

\section{RESULTS}

\section{Generation of ATM1 and ATM2 YFP-Fusion Constructs}

Alignment of the amino acid sequences of the four class VIII myosins from A. thaliana resulted in a cladogram grouping AT3G19960 (ATM1) together with AT1G50360 and AT5G54280 (ATM2) together with AT4G27370 (Fig. 1A). In Figure 1B, the alignment of the amino acid sequences of the respective globular tail domains, starting just behind the IQ repeats, illustrates sequence identities and similarities between the four $A$. thaliana myosin VIII proteins. According to publicly available maps of the tissue- and developmental-specific expression of these myosins (http://bbc.botany.utoronto.ca/efp/cgi-bin/efpWeb.cgi), ATM1 and ATM2 occur essentially throughout the whole plant at all developmental stages, except in pollen. The expression levels in roots are low for ATM1 and not detectable for ATM2. AT4G27370 is exclusively pollen specific and for AT1G50360 a detailed expression map is not available 


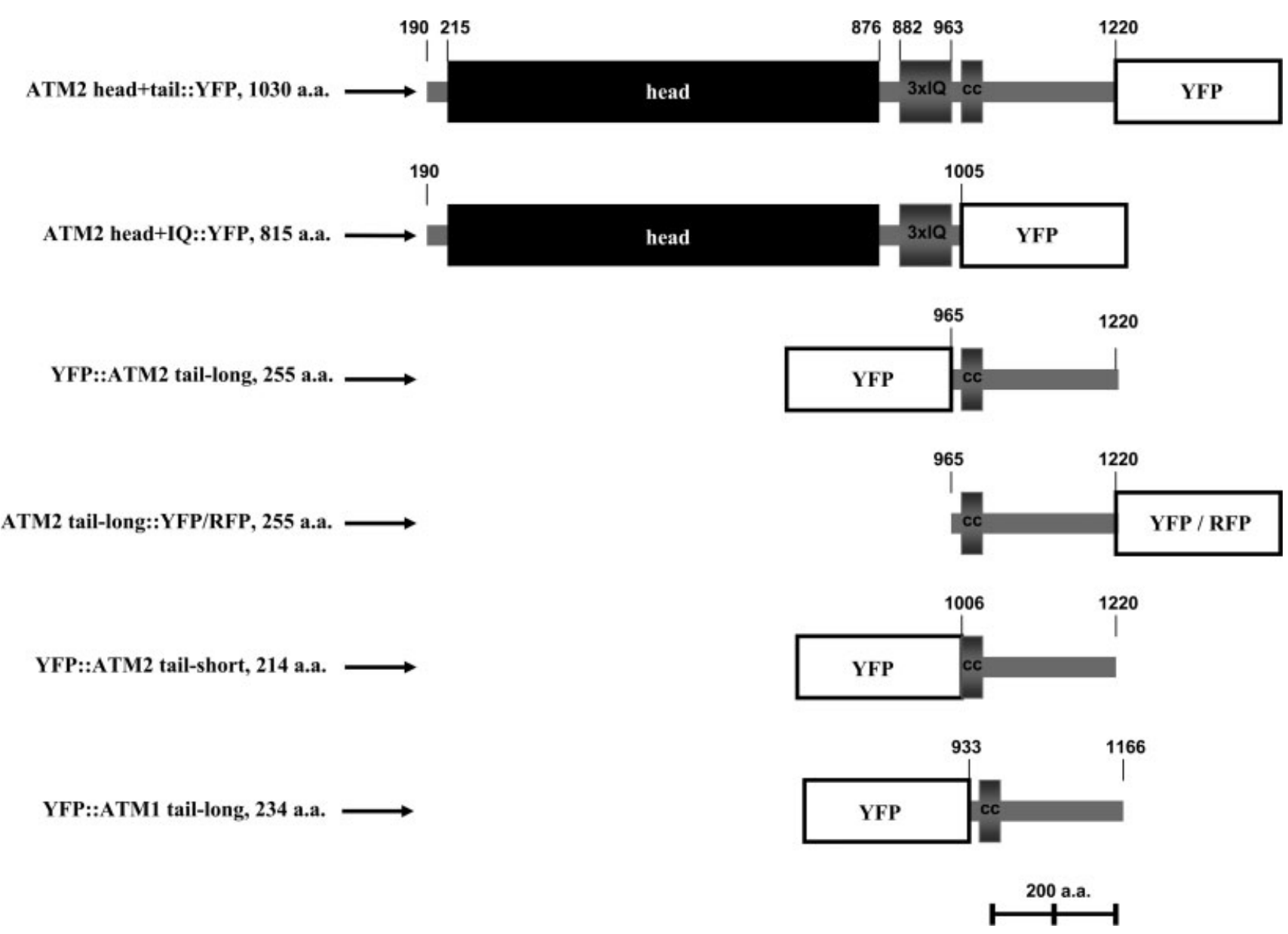

Fig. 2. Schematic representation of ATM2 and ATM1 fluorescent protein fusion constructs. cDNA fragments of the Arabidopsis thaliana class VIII myosins, ATM1 and ATM2, were fused in frame with the YFP or RFP cDNA at the C-terminal or N-terminal end. The positions of the amino acids based on the full length protein sequence is shown for each construct.

yet, however it appears to be expressed in green tissue parts. Because of their presence in almost all organs and tissues of $A$. thaliana we investigated the subcellular localization of ATM1 and ATM2. For this purpose, a number of YFP-fusion constructs with the respective cDNA fragments have been designed (Fig. 2) for use in transient expression in $N$. benthamiana (A. tumefaciens-mediated transformation). Regarding ATM2, five constructs were made: a nearly full-length construct with C-terminal YFP fusion (ATM2 head+tail::YFP), a construct containing the head and neck domain with Cterminal YFP fusion (ATM2 head+IQ::YFP), two YFP tail domain fusions starting just behind the neck domain with YFP (RFP) fused either to the N-terminus (YFP::ATM2 tail-long) or to the C-terminus (ATM2 taillong::YFP/RFP) and a slightly shorter tail construct starting just with the coiled-coil domain fused $\mathrm{N}$-terminally with YFP (YFP::ATM2 tail-short). For ATM1 only one construct was generated which was comprised of the full- length tail domain, starting just behind the neck domain, fused N-terminally with YFP (YFP::ATM1 tail-long).

\section{Transient Expression of ATM2 Fusion Proteins}

Between 3 and 5 days after inoculation of $N$. benthamiana leaves with A.tumefaciens, the YFP fluorescence was recorded with CLSM in epidermal cells. In Figure 3, the subcellular patterns of the various YFPtagged ATM2 proteins are displayed. ATM2 head+ tail::YFP labeled filamentous structures with some vesicles along them (Fig. 3A). We also tried to express this ATM2 head+tail fragment as an N-terminal YFP fusion, but this resulted in only weak cytoplasmic signals without distinct localization (not shown). The ATM2 head+IQ::YFP protein exclusively decorated filaments throughout the whole cell, probably because of the actin binding site of the head domain (Fig. 3B). In contrast, all the three ATM2 tail YFP fusions labeled vesicles, although the pattern and appearance of these vesicles varyied (Figs. 

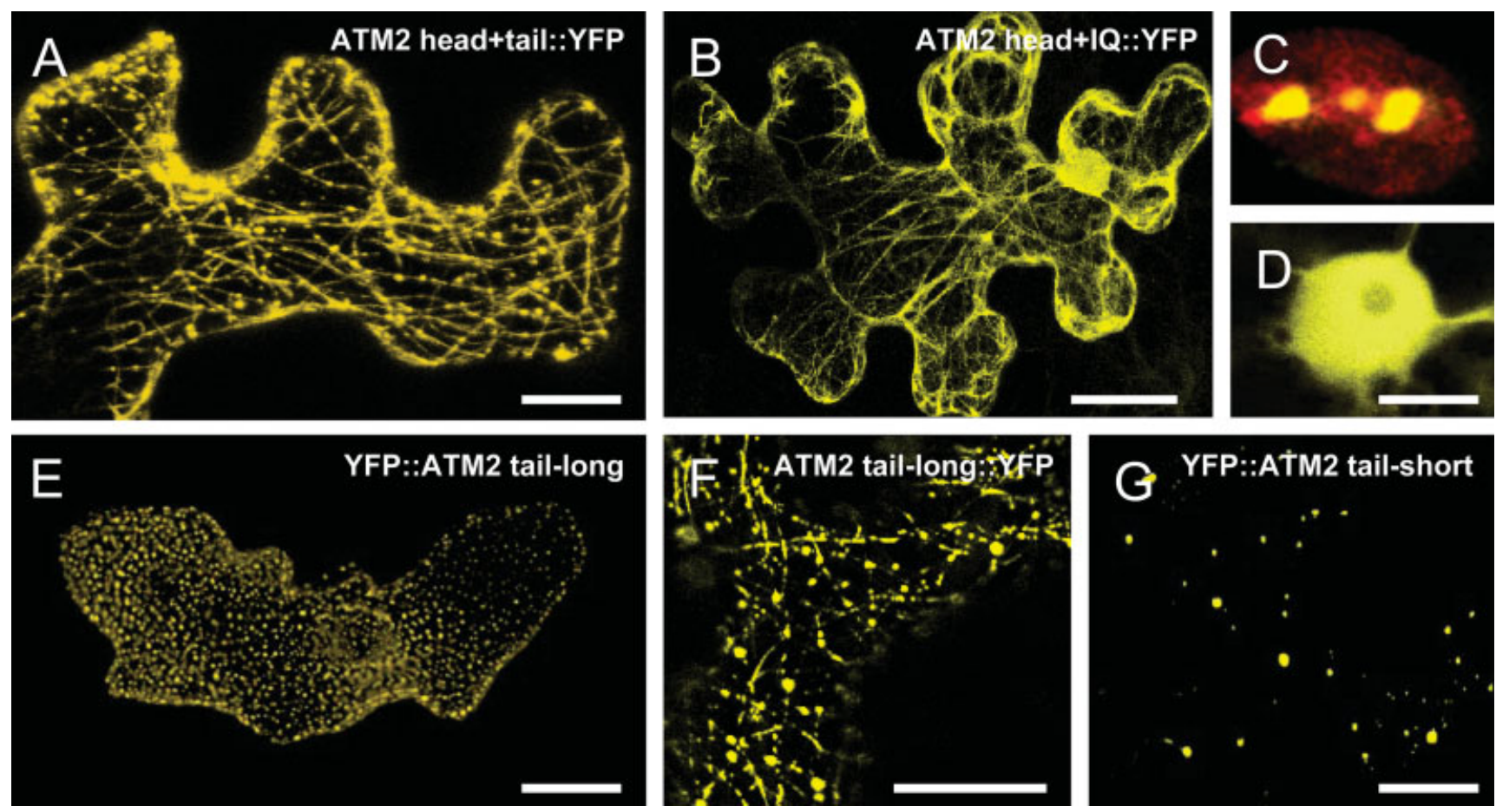

Fig. 3. Subcellular localization of ATM2 fusion proteins. A number of ATM2 fragments differing in length and domains were fused at the C-terminal or N-terminal end with YFP. The constructs were transiently expressed in $N$. benthamiana leaves. Recording of images was by CLSM of epidermal cells. A: ATM2 head + tail::YFP. B: ATM2 head-

IQ::YFP. C: YFP::ATM2 tail-long, nuclear counterstain with propidium iodide. D: control with YFP alone. E: YFP::ATM2 tail-long. F: ATM2 tail-long::YFP. G: YFP::ATM2 tail-short. Images in A, B, D, and $\mathrm{E}$ are $3 \mathrm{D}$ projections from 20 optical sections. The bar in $\mathrm{A}, \mathrm{B}$, and E corresponds to $100 \mu \mathrm{m}$, in D to $5 \mu \mathrm{m}, \mathrm{F}$ to $50 \mu \mathrm{m}$, and in $\mathrm{G}$ to $80 \mu \mathrm{m}$.

3D-3F). The prevailing vesicular pattern (in $80 \%$ of cells) of those three fusion constructs was as follows: ATM2 tail-long::YFP labeled vesicles in rows along filaments (Fig. 3E), YFP::ATM2 tail-long visualized densely packed small vesicles of similar diameter (about $1 \mu \mathrm{m}$ ) in the cell periphery (Fig. 3D) and YFP::ATM2 tail-short labeled vesicular structures of variable diameter $(0.5-4 \mu \mathrm{m})$ which, in contrast to the others, were motile (Fig. 3F). All ATM2 tail constructs labeled 1-3 distinct dots of variable size within nuclei, as illustrated in Figure $3 \mathrm{C}$ for YFP::ATM2 tail-long employing nuclear counterstaining with propidium iodide. The appearance of this subnuclear localization pattern was distinct from the nuclear labeling with YFP alone which was evenly distributed throughout the whole nucleus except the nucleolus (Fig. 3D).

\section{Coexpression of ATM2 Tail Constructs}

In order to examine the difference between the vesicular localization patterns obtained with the three ATM2 tails, coexpression studies were performed using ATM2 tail-long::RFP together with YFP::ATM2 tailshort (Figs. 4A-4C) and ATM2 tail-long::RFP together with YFP::ATM2 tail-long (Figs. 4D-4F). While the pattern of the single-expressed ATM2 tail-long::YFP was "vesicles in a row" and of the single-expressed
YFP::ATM2 tail-short variable-sized motile vesicles (see Figs. 3D and 3F), coexpression resulted in colocalization of the differently tagged vesicles (Figs. 4A-4C). Similarly, extensive colocalization, at least with respect to all larger vesicles, was found upon coexpression of ATM2 tail-long::RFP and YFP::ATM2 tail-long (Figs. 4D-4F), although single expression of the latter revealed densely packed small peripheral vesicles. Thus, by coexpressing the different ATM2 tail versions, the patterns of labeled vesicular structures merged. This indicates a close interrelation of the vesicular targets.

\section{Colocalization of ATM2 With Endosomes}

To identify the nature of the vesicles that are the targets of ATM2, comprehensive colocalization studies were carried out. An array of fluorescence protein-tagged markers for organelles such as peroxisomes, Golgi vesicles, ER and mitochondria was employed in coexpression experiments with the ATM2 tail constructs. No colocalization of the organelles with the ATM2 tail YFP-fusion proteins was found (data not shown). Further experiments revealed that coexpression of YFP::ATM2 tail-long with the endosomal marker FYVE resulted in partial colocalization (Figs. 5A-5C). The double-labeled vesicles were motile and small- to medium-sized (1.5-4 

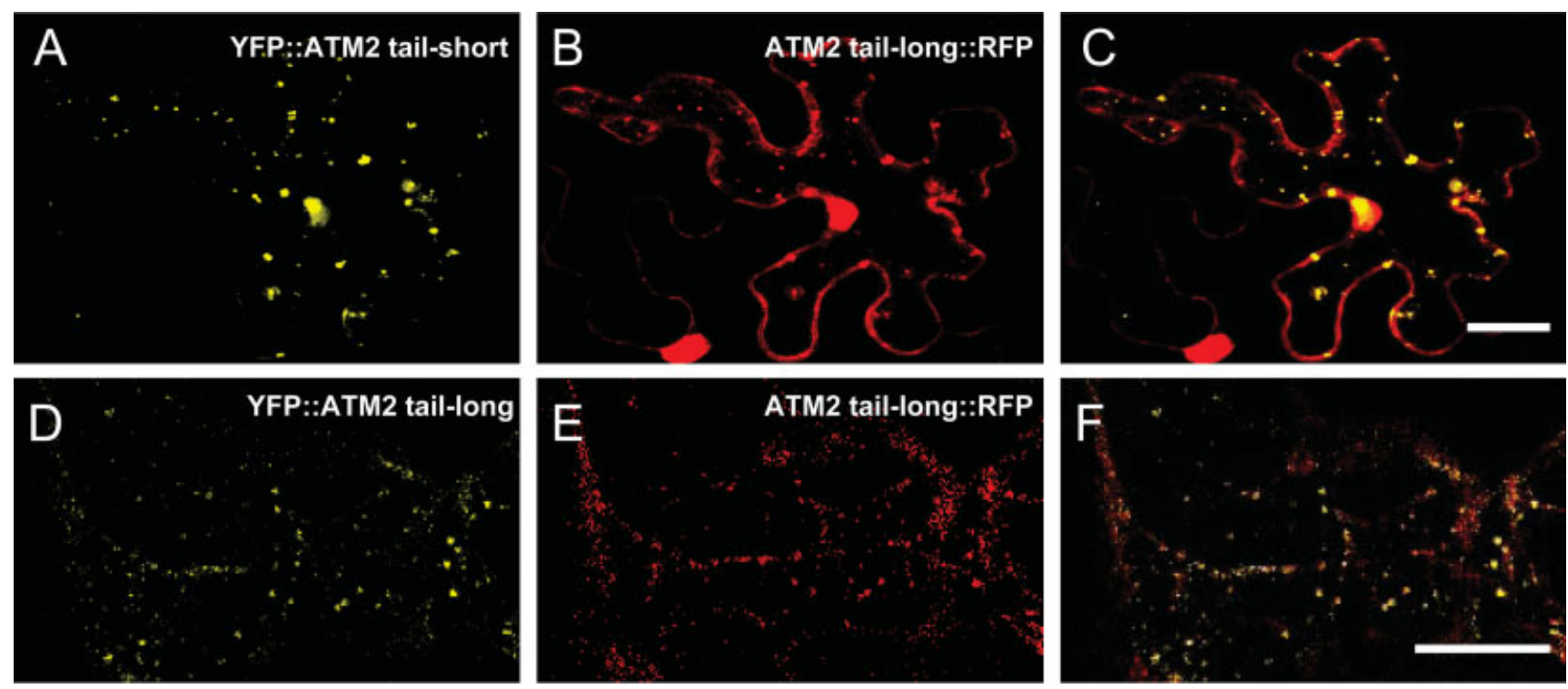

Fig. 4. Coexpression of ATM2 tail constructs. The three ATM2 tails, ATM2 tail-long::RFP, YFP::ATM2 tail-short, and YFP::ATM2 tail-long were assessed for colocalization in epidermal cells of $N$. benthamiana by using CLSM. A-C: Coexpression of YFP::ATM2 tail-short (A) and ATM2 taillong::RFP (B). D-F: Coexpression of YFP::ATM2 tail-long (D) and ATM2 tail-long::RFP (E). (C,F) Respective merged images. The bar in A-C coresponds to $150 \mu \mathrm{m}$, in D-F to $30 \mu \mathrm{m}$.

$\mu \mathrm{m}$ in diameter), while the bulk of the small peripheral, non-motile vesicles were not labeled by the FYVE marker.

In a further set of experiments, the dye FM4-64, which is widely used to study endocytosis, was applied to $N$. benthamiana leaf tissue expressing either the YFP::ATM2 tail-long or the YFP::ATM2 tail-short. At various times after dye treatment, tissue was imaged by CLSM. Already after 15 min of preincubation with a FM4-64 solution, the internalized dye stained vesicles in epidermal cells. Importantly, two different types of vesicles were labeled by FM4-64 (Figs. 5D-5F). In cells in which the YFP-fusion protein was expressed, small peripheral vesicles showed YFP labeling (Fig. 5D). In these cells the same vesicles were also stained with FM4-64 (Figs. 5E and 5F). In contrast, cells lacking expression of YFP::ATM2 tail-long displayed FM4-64 labeling of larger and motile vesicles that were variable in size $(2-5 \mu \mathrm{m}$ in diameter) and sometimes irregular in shape (Figs. 5E and 5F). The appearance of the YFP and FM4-64 double-labeled small vesicles essentially did not change for at least $1 \mathrm{~h}$. In cells expressing YFP::ATM2 tail-short, nearly $100 \%$ double-labeling of YFP and FM4-64 of the motile, variable sized vesicles, which are typically seen by labeling with this fusion protein (see Fig. 3), was observed (Figs. 5G-5I).

To test whether endosomes containing the internalized receptor BRI1 actually bind to ATM2, the colocalization of CFP::BRI1 with YFP::ATM2 tail-long was examined. Upon coexpression of these constructs in $N$. benthami- ana leaves, vesicles were detected that showed labeling with both of the FP-fusion proteins (Figs. 5K-5M).

\section{Plasmodesmatal Targeting of the ATM1- and ATM2-Tail Domain}

Occasionally, in about $10 \%$ of cells, expression of the YFP::ATM2 tail-long lead to a localization at the wall of epidermal cells (Figs. 5A and 5B). It localized to spots opposite to each other at either side of the wall between two adjacent epidermal cells. When the tissue was plasmolyzed, cells with high expression levels of YFP::ATM2 tail-long showed labeling of Hecht threads and, strikingly, fluorescent spots that were not retracted during the process of plasmolysis and remained at the cell wall (Figs. 5E$5 \mathrm{G})$. The well-known Hecht threads are formed upon plasmolysis because of the continuation of the plasma membrane into the adjacent cell through plasmodesmata. Thus the YFP::ATM2 tail-long labeled spots at the wall of plasmolysed cells could mark sites of plasmodesmata. As a control for this subcellular targeting of the ATM2 tail fusion protein in $N$. benthamiana epidermal cells the YFP-tagged ATM1 tail domain (YFP::ATM1 tail-long) was expressed which resulted in a localization pattern very similar to YFP::ATM2 tail-long (Figs. 5C and 5D).

\section{DISCUSSION}

We have used confocal laser scanning microscopy in combination with transient expression of tailor-made 

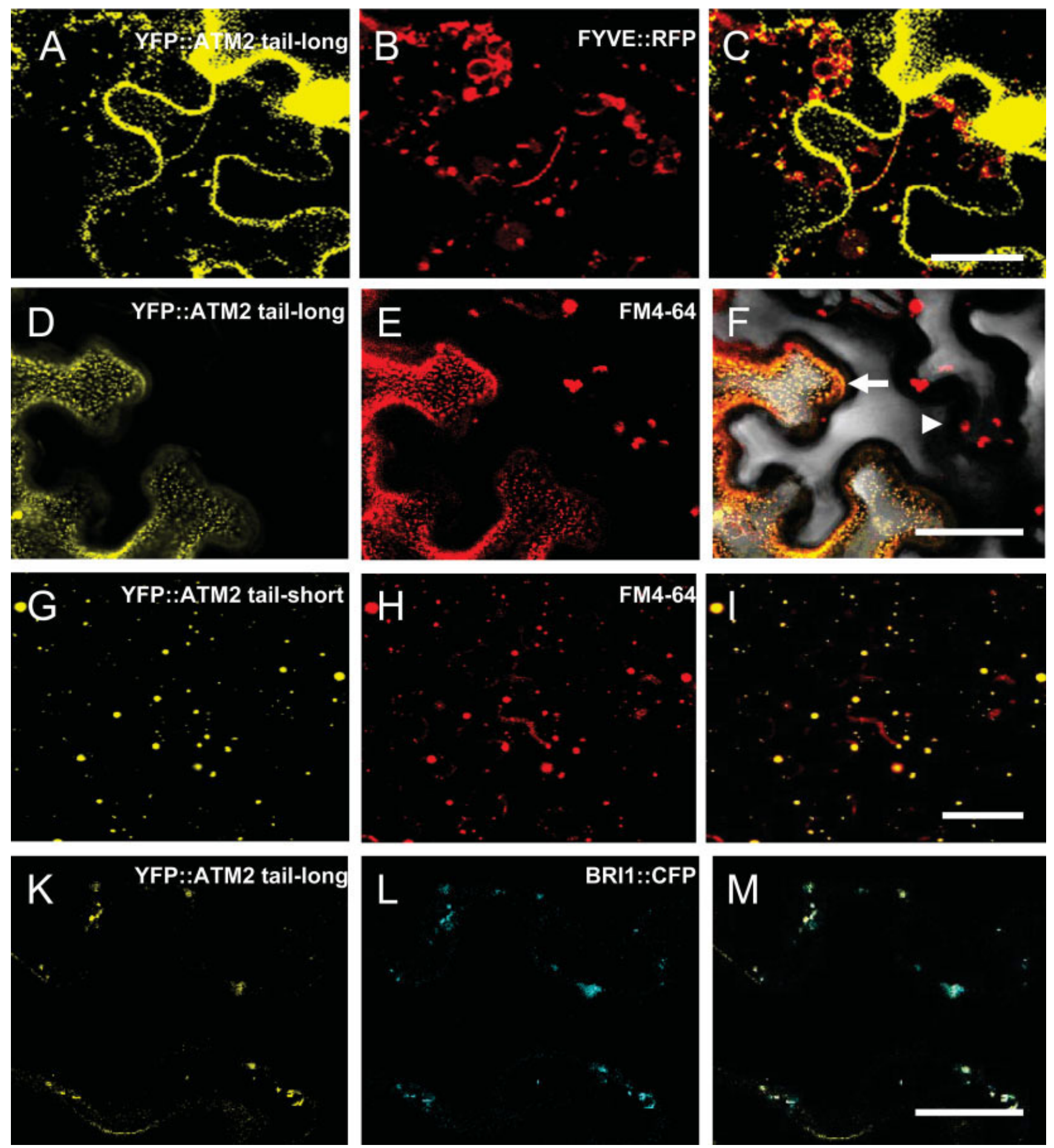

Fig. 5. Colocalization of the ATM2 tail with endosomal markers and the brassinosteroid receptor BRI1. Coexpression of (A) YFP::ATM2 taillong and (B) FYVE-dsRed tagged endosomal marker, (C) merged image. $N$. bentamiana leaves transiently expressing (D) YFP::ATM2 tail-long and (G) YFP::ATM2 tail-short were treated with FM4-64. E,H: staining with FM4-64. F: merged fluorescent and brightfield image, arrow is pointing to a cell expressing YFP::ATM2 tail-long colocalizing with FM4-64, arrow head is pointing to a cell not expressing YFP::ATM2 taillong and showing FM4-64 staining of larger vesicles. I: Merged fluorescence image. K: Vesicular labeling with YFP::ATM2 tail-long. L: Labeling of vesicles with BRI1-CFP. M: Merged image. The bar corresponds to $20 \mu \mathrm{m}$ in $\mathrm{C}$ and $\mathrm{M}$ and to 40 and $50 \mu \mathrm{m}$ in $\mathrm{F}$ and I, respectively. 

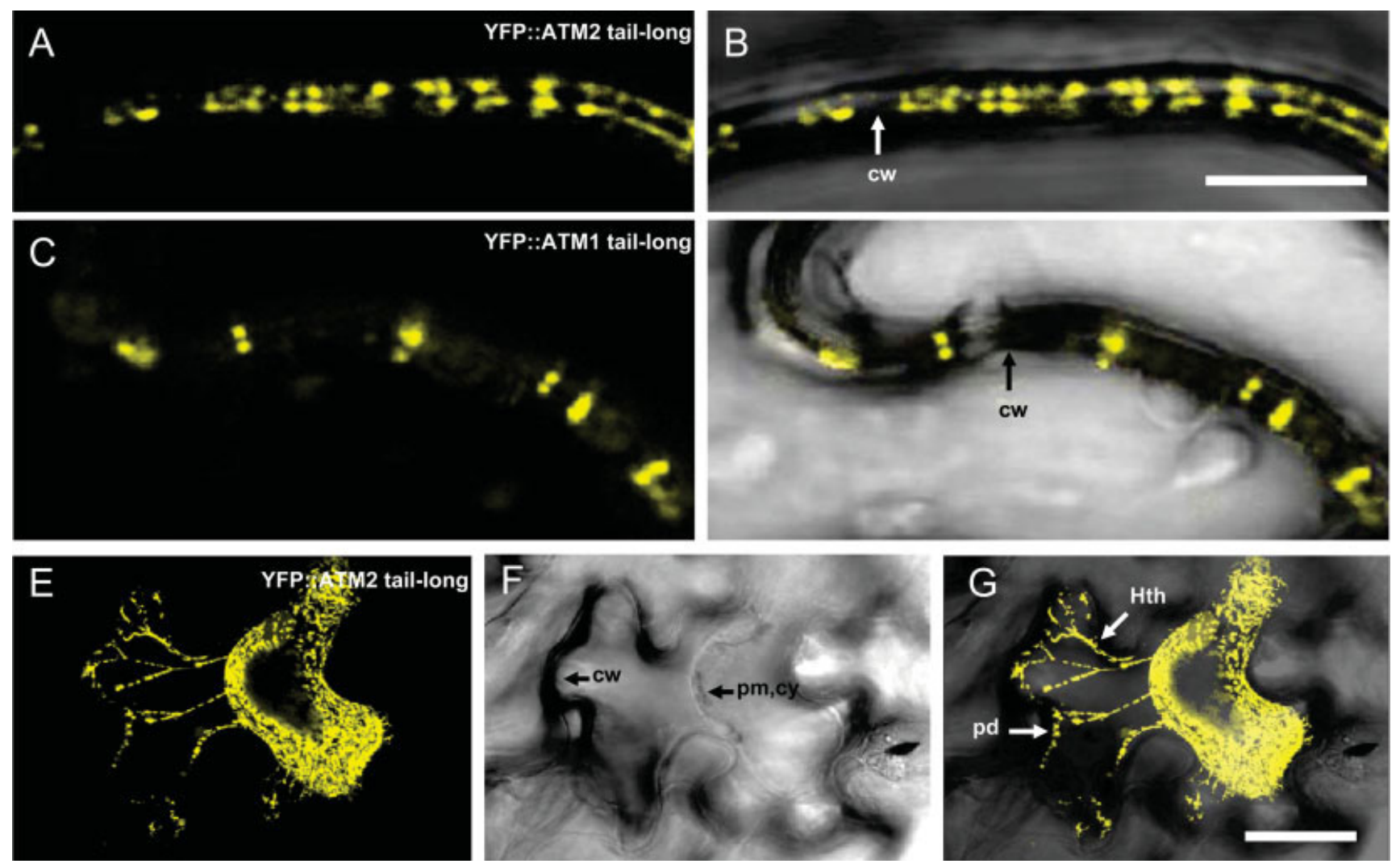

Fig. 6. Plasmodesmatal targeting of ATM1 and ATM2 tails. The full length tail domains of ATM1 and ATM2 fused N-terminally toYFP were expressed transiently in epidermal cells of $N$. benthamiana. The subcellular localization was monitored with CLSM. A,B: YFP::ATM1 tail; fluorescent image (A), merged fluorescent and brightfield image (B). C,D: YFP::ATM2 tail-long; fluorescent image (C), merged fluorescent and brightfield image (D). E,F: Localization of YFP::ATM2 tail-long upon plasmolysis; fluorescent image (E), brightfield image (F), merged fluorescent and brightfield image $(\mathbf{G})$. Arrows in B and D point to the cell wall, in $\mathrm{F}$ to the retracted plasma membrane and cytoplasm; in $\mathrm{G}$ to Hecht threads and plasmodesmata. cw: cell wall, cy: cytoplasm, Hth: Hecht threads, pd: plasmodesmata, pm: plasma membrane. The bar corresponds to $10 \mu \mathrm{m}$ in $\mathrm{A}-\mathrm{D}$, to $50 \mu \mathrm{m}$ in $\mathrm{E}-\mathrm{F}$. [Color figure can be viewed in the online issue, which is available at www.interscience.wiley.com.]
A. thaliana class VIII myosin cDNA constructs fused to genetically encoded fluorescent protein tags for the in vivo determination of cargo. Such an in vivo imaging approach was already successfully applied for cargo identification of class XI myosins from A. thaliana $[\mathrm{Li}$ and Nebenfuhr, 2007; Reisen and Hanson, 2007]. These data have clearly demonstrated that transient expression of myosin cDNA fragments from A. thaliana fused to cDNA of fluorescent proteins results in binding to the same organelles in A. thaliana, $N$. benthamiana and Allium cepa (onion). This strongly indicates conservation of respective structural features and the underlying binding machinery. Because of the high transformation rate and the expression window of 3-5 days, we have preferentially used the A.tumefaciens based transformation of $N$. benthamiana. When YFP::ATM2 tail-long and YFP::ATM1 tail were expressed in epidermal cells of $A$. thaliana leaves upon biolistic transformation, the binding specificities were the same as in $N$. benthamiana, i.e., small, non-motile peripheral vesicles and plasmodesmata association, respectively (data not shown).

\section{Different Localization Patterns of Fluorescent Protein-Tagged ATM2 Proteins}

The head and tail domain of myosins have different functions. The head domain is responsible for force production and movement and binds to actin filaments, whereas the tail domain binds to cargo. It is possible that the binding to actin filaments somehow prevailed when the nearly full-length ATM2 head+tail::YFP construct was expressed (Fig. 3), since the C-terminally fused YFP molecule might have hindered efficient cargo-binding. The fact that all ATM2 tail constructs labeled vesicles and, on the other hand, the ATM2 head domain (ATM2 head + IQ::YFP) labeled filaments exclusively, is consistent with the idea of different tasks of the head and tail domain. Expression of the different ATM2 tail YFP fusions resulted in varying localization patterns (Fig. 3), 
although we observed preferences: the full-length N-terminal tail YFP-fusion (YFP::ATM2 tail-long) labeled small, densely packed, immotile vesicles within the cell periphery, while the C-terminal YFP-fusion of this construct decorated immotile small vesicles along filaments like beads on a string. Surprisingly, the slightly shorter tail domain (YFP::ATM2 tail-short) was found on motile vesicles. However, in coexpression experiments, these different patterns somehow converged (Fig. 4). This observation suggests that the tail domain of ATM2 probably possesses binding capability to one vesicular cargo only. The variation in the localization pattern of the three constructs might have several reasons. First of all, overexpression of the fusion proteins might result in dominant negative interference with the function(s) of the endogenous, native myosin ATM2. Dominant negative inhibition of transport processes by ectopic overexpression of myosin tail domains has been clearly demonstrated for class XI myosins [Avisar et al., 2008b] as well as for class VIII myosins [Avisar et al., 2008a] and was actually taken as experimental proof for the requirement of the respective myosins in organelle trafficking and plasmodesmatal targeting. Since the expression levels in individual epidermal cells in our experiments certainly vary, the dominant negative inhibition might be different. Furthermore, it can not be ruled out that the strength of cargo binding of the fusion proteins is affected by their length and by the positioning of the fluorescence tag. Consequently, the competition with binding sites of the native protein might be subjected to variation. The degree of the dominant negative effect can vary between experiments and cells. Assuming the native ATM2 is involved in endocytosis, differences in the actual strength of dominant negative inhibition might affect the proportional composition of the various vesicles in this pathway by partly interrupting or at least slowing down the native vesicular flow. This might then finally be reflected by the observed variation in localization patterns of the ATM2 fusion proteins.

Since all our tail constructs possessed the coiledcoil region they potentially would have been able to interact with each other or even with the native ATM2. The formation of such irregular dimers upon coexpression of different constructs might contribute to the merging of localization patterns.

Surprisingly the tail domains of ATM2 were also found within nuclei (Figs. 4C and 4D). The observed vesicular bodies might represent a sort of deposit due to overexpression of the fusion proteins of ATM2tail; however, the specific localization in the nucleus might indicate a specific nuclear function for ATM2. In mouse cells it was demonstrated that an isoform of myosin I is localized in the nucleus and colocalized with RNA polymerase II [Pestic-Dragovich et al., 2000] suggesting participation in transcription.

\section{Involvement of ATM2 in Endocytosis}

Class VIII myosins have been proposed to be the motors for endocytosis in plant cells [Baluska et al., 2004]. Results from pharmacological studies pointed in this direction [Baluska et al., 2004; Samaj et al., 2005]. Furthermore, motility of endosomes is actin-dependent, according to experiments in which the GFP/RFP FYVE construct was used as a reporter in transgenic plants of A. thaliana and Medicago truncatulata in tip-growing root hairs. The identity of the FYVE-labeled endosomes was confirmed in vivo using GFP/YFP-tagged constructs of the endosome-specific plant Rab GTPases Ara6 and RabF2a and by the endocytic tracer FM4-64 [Voigt et al., 2005]. In our study, direct evidence was obtained for the participation of the class VIII myosin ATM2 in endocytosis. The N-terminal YFP fusion of the ATM2 tail domain (YFP::ATM2 tail-long) displayed partial colocalization with the endosomal RFP-fluorescent marker FYVE (Figs. 5A-5C). The rapidly moving vesicles carrying both tags, YFP::ATM2 tail-long and RFP-FYVE, were preferentially observed in cells possessing lower expression levels. It is possible that the small, immotile vesicles seen in cells with high expression levels are at a very early stage of the endocytotic pathway and thus escaped from tagging by the FYVE marker. In animal cells, myosin VI has an important function in endocytosis. It binds to early endosomes and facilitates further transportation through the endocytotic pathway [Buss et al., 2001; Aschenbrenner et al., 2003]. Overexpression of the tail domain of myosin VI causes a dominant negative inhibition of this pathway. The early endosomes are not processed further and accumulate in the cell periphery. This resembles the picture after overexpression of the ATM2 tail- $\mathrm{N}$-terminal YFP-fusion (YFP::ATM2 tail-long) in $N$. benthamiana and in A. thaliana cells, namely the occurrence of the bulk of YFP-labeled small, immotile peripheral vesicles (Fig. 5D). When the widely used endosomal tracer FM4-64 was applied, precisely these vesicles were labeled in cells expressing YFP::ATM2 tail-long (Fig. 5E). In cells expressing YFP::ATM2 tail-short, however, the resulting YFP-tagged motile vesicles were stained by the internalized FM4-64 dye (Figs. 5G-5I). This supports the endosomal identity of both sorts of vesicles. Finally, coexpression of the constitutively internalized membrane receptor BRI1 [Russinova et al., 2004] as CFP fusionprotein with YFP::ATM2 tail-long resulted in doublelabeling of vesicles demonstrating again binding of the ATM2 tail to endosomes (Figs. 5K-5M). Interestingly, in a recent publication the tail domain of ATM1 was also found to colocalize with internalized FM4-64 and partially with the endosomal marker ARA6. In rare cases colocalization with the other markers ARA7 and FYVE was observed [Golomb et al., 2008]. The authors suggest 
from these results an involvement of class VIII myosins, and particularly of ATM1, in endocytosis.

\section{Association of ATM1 and ATM2 With Sites of Plasmodesmata}

Using ATM1-specific antibodies it has been demonstrated that in the apices of maize roots sites of plasmodesmata were labeled along the plasma membrane at newly forming cell walls [Reichelt et al., 1999; Wojtaszek et al., 2005]. The pattern that we observed in mature epidermal cells by overexpression of YFP::ATM1-taillong in $N$. benthamiana epidermal leaf cells is in agreement with this previous finding (Fig. 6). The observation, that YFP::ATM2 tail-long is localized occasionally at similar sites, suggests participation in transport processes towards plasmodesmata, perhaps, in combination with ATM1. Very recently, three $N$. benthamiana orthologs of the A. thaliana class VIII myosins ATM1, ATM2 and ATMB were characterized and shown to be involved in plasmodesmatal targeting [Avisar et al., 2008a]. Furthermore, in another recent publication the tail domain of ATM1 was localized in pit fields accumulating callose and, very remarkably, colocalized with the tail domain of ATM2 at specific sites of the plasma membrane upon transient expression in $N$. benthamiana leaves [Golomb et al., 2008]. Thus, ATM2 might play a role, though with differential preference, in endocytosis and plasmodesmatal targeting.

\section{CONCLUSIONS}

In analogy to the concept of endocytosis in animals and yeast, regulated vesicle trafficking to and from the cell surface probably controls the levels and activities of receptors, transporters and other plasma membrane proteins in plants. This analogy also could hold true for the involvement of myosins in endocytosis as is suggested for animal cells [Buss et al., 2001; Aschenbrenner et al., 2003; Holt et al., 2007]. Accordingly, as our results indicate, the class VIII myosin ATM2 binds to early endosomes and mediate transportation through the endocytic pathway. Other recent data suggest, that ATM2 could act in concert with ATM1 in this respect [Golomb et al., 2008]. In addition, our and other results indicate that the members of class VIII myosins could also cooperate in plasmodesmatal targeting [Avisar et al., 2008a; Golomb et al., 2008]. Hence, it is conceivable that the two classes of higher plant myosins possess differential functions. The class XI members might be essentially required for the transportation of organelles such as Golgi stacks, peroxisomes and mitochondria whereas class VIII members are involved in endocytosis and plasmodesmatal targeting. Further studies have to investigate the detailed sequence of events in plant receptor internalization and the participation of ATM2 and other myosins in endocytic vesicle trafficking and the process of material delivery to plasmodesmata.

\section{ACKNOWLEDGMENTS}

The authors thank B. Voigt and F. Baluska for providing us with the tandem RFP-FYVE marker construct and S.C. de Vries for the BRI1-CFP plasmid.

\section{REFERENCES}

Aschenbrenner L, Lee T-T, Hasson T. 2003. Myo6 facilitates the translocation of endocytic vesicles from cell peripheries. Mol Biol Cell 14:2728-2743.

Avisar D, Prokhnevsky AI, Dolja VV. 2008a. Class VIII myosins are required for plasmodesmatal localization of a closteroviral Hsp70 homolog. J Virol DOI:10.1128/JVI 02246-07.

Avisar D, Prokhnevsky AI, Makarova KS, Koonin EV, Dolja VV. 2008b. Myosin XI-K is required for rapid trafficking of Golgi stacks, peroxisomes and mitochondria in leaf cells of Nicotiana benthamiana. Plant Physiol DOI:10.1104/pp. 107.113647.

Baluska F, Samaj J, Hlavacka A, Kendrick-Jones J, Volkmann D. 2004. Actin-dependent fluid-phase endocytosis in inner cortex cells of maize root apices. J Exp Bot 55:465-473.

Berg JS, Powell BC, Cheney RE. 2001. A millennial myosin census. Mol Biol Cell 12:780-794.

Buss F, Arden SD, Lindsay M, Luzio JP, Kendrick-Jones J. 2001. Myosin VI isoform localized to clathrin-coated vesicles with a role in clathrin-mediated endocytosis. EMBO J 20:3676-3684.

Geldner N. 2004. The plant endosomal system-its structure and role in signal transduction and plant development. Planta 219:547-560.

Gifford ML, Robertson FC, Soares DC, Ingram GC. 2005. Arabidopsis CRINCLY4 function, internalization, and turnover are dependent on the extracellular crinkly repeat domain. Plant Cell 17:1154-1166.

Golomb L, Abu-Abied M, Belausov E, Sadot E. 2008. Different subcellular localizations and functions of Arabidopsis myosin VIII. BMC Plant Biol 8:doi:10.1186/1417-2229-8-3.

Gomez-Gomez L, Boller T. 2002. Flagellin perception: A paradigm for innate immunity. Tr Plant Sci 7:251-256.

Hashimoto K, Igarashi H, Mano S, Nishimura M, Shimmen T, Yokota E. 2005. Peroxisomal localization of a myosin XI isoform in Arabidopsis thaliana. Plant Cell Physiol 46:782-789.

Hodge T, Cope MJ. 2000. A myosin family tree. J Cell Sci 113:33533354.

Holt JP, Bottomly K, Mooseker MS. 2007. Assessment of myosin II, $\mathrm{Va}$, and VIIa loss of function on endocytosis and endocytic vesicle motility in bone marrow-derived dendritic cells. Cell Motil Cytoskel 64:765-766.

Holweg C, Nick P. 2004. Arabidopsis myosin XI mutant is defective in organelle movement and polar auxin transport. Proc Natl Acad Sci USA 101:10488-10493.

Koncz C, Mayerhofer R, Koncz-Kalman C, Nawrath C, Reiss B, Redei G, Schell J. 1990. Isolation of a gene encoding a novel chloroplast protein by T-DNA tagging in Arabidopsis thaliana. EMBO J 9:1337-1346.

Li J-F, Nebenfuhr A. 2007. Organelle targeting of myosin XI is mediated by two globular tail subdomains with seperate cargo binding sites. J Biol Chem 282:20593-20602.

Peremyslov VV, Prokhnevsky AI, Avisar D, Dolja VV. 2008. Two class XI myosins function in organelle trafficking and root hair development in Arabidopsis thaliana. Plant Physio. 1107: 113654, DOI:10.1104/pp. 
Pestic-Dragovich L, Stojiljkovic L, Philimonenko A, Nowak G, Ke Y, Settlage R, Shabanowitz J, Hunt D, Hozak P, Lanerolle P. 2000. A myosin I isoform in the nucleus. Science 290:337341.

Reddy ASN, Day IS. 2001. Analysis of the myosins encoded in the recently completed Arabidopsis thaliana genome sequence. Genome Biol 2:0024.1-0024.17.

Reichelt S, Knight AE, Hodge TP, Baluska F, Samaj J, Volkmann D, Kendrick-Jones J. 1999. Characterization of the unconventional myosin VIII in plant cells and its localization at the post-cytokinetic cell wall. Plant J 19:555-567.

Reisen D, Hanson MR. 2007. Association of six YFP-myosin XI-tail fusions with mobile plant cell organelles. BMC Plant Biol 7:117.

Robatzek S, Chinchilla D, Boller T. 2006. Ligand-induced endocytosis of the pattern recognition receptor FLS2 in Arabidopsis. Genes Dev 20:537-542.

Russinova E, Borst J-W, Kwaaitaal M, Cano-Delgado A, Yin Y, Chory J, de Vries SC. 2004. Heterodimerization and endocyto- sis of Arabidopsis brassinosteroid receptors BRI1 and AtSERK3 (BAK1). Plant Cell 16:3216-3229.

Samaj J, Read ND, Volkmann D, Menzel D, Baluska F. 2005. The endocytic network in plants. Tr Cell Biol 15:425-433.

Voigt B, Timmers ACJ, Samaj J, Hlavacka A, Ueda T, Preuss M, Nielsen E, Mathur J, Emans N, Stenmark H. 2005. Actin-based motility of endosomes is linked to the polar tip growth of root hairs. Eur J Cell Biol 84:609-621.

Voinnet O, Rivas S, Mestre P, Baulcombe D. 2003. An enhanced transient expression system in plants based on suppression of gene silencing by the $\mathrm{p} 19$ protein of tomato bushy stunt virus. Plant J 33:949-956.

Wang Z, Pesacreta TC. 2004. A subclass of myosin XI is associated with mitochondria, plastids and the molecular chaperone subunit TCP-1a in maize. Cell Motil Cytoskeleton 57:218-232.

Wojtaszek P, Anielska-Mazur A, Gabrys H, Baluska F, Volkmann D. 2005. Recruitment of myosin VIII towards plastid surfaces is root-cap specific and provides the evidence for actomyosisn involvement in root osmosensing. Funct Plant Biol 32:721-736. 